\title{
ERRATUM
}

\section{D'Arcy Thompson and the theory of transformations}

\section{Wallace Arthur}

Nature Reviews Genetics 7, 401-406 (2006), doi:10.1038/nrg1835

The two photos published in Figure 1 of this article were reproduced with permission from Oxford University Press, which published the images. The original prints are in fact held with the Papers of D'Arcy Wentworth Thompson and have been reproduced courtesy of the University of St Andrews Library, to which we are grateful. 